\title{
Data Fusion Analysis based Active Thermography for SNR Enhancement
}

\author{
by Wontae $\mathrm{Kim}^{1, \dagger}$, Ranjit Shrestha ${ }^{1}$, and Manyong $\mathrm{Choi}^{2}$
}

${ }^{1}$ Department of Mechanical Engineering, Kongju National University, 1223-24, Cheonan-daero, Seobuk-gu, Cheonan-si, Chungcheongnam-do 31080, Republic of Korea, ${ }^{\dagger} k w t @ k o n g j u . a c . k r$, sthranjit@gmail.com

${ }^{2}$ Safety Measurement Center, Korea Research Institute of Standards and Science, PO Box 102, Yuseong-gu, Daejeon, 305-600, Republic of Korea, mychoi@kriss.re.kr

\begin{abstract}
Active thermography is an emerging technique for non-destructive testing and evaluation of a variety of materials, structures, and components due to its non-contact, whole-field, high speed and qualitative and quantitative inspection capabilities. Fourier transform allows for the computation of amplitude and phase angle images from the temperature-time history of each pixel. Amplitude image focuses on the surface of a sample up to a limited depth and is suitable to extend the object surface shape information. Phase image is less sensitive to shape information; independent of local disturbances such as non-uniform heating and emissivity; influenced by the depth attained by the thermal wave and is suited for computation of defect depth. This paper proposes a Wavelet-based data fusion method to combine the details obtained from the Fourier transform for Lock-in thermography experiment carried over Stainless steel sample and compares the input Amplitude and Phase images and output fusion image with respect to signal to noise ratio.
\end{abstract}

\section{Introduction}

Active thermography integrates infrared imaging with external heating and has proved to be an essential approach for non-destructive testing and evaluation (NDT\&E) of the structural integrity of materials due to its inherent merits such as non-contact, high speed, whole-field, qualitative and quantitative inspection capabilities. Fourier transform has been used by many scholars to evaluate the temperature response in the frequency domain. Fourier transforms allows for the computation of amplitude and phase angle data from the temperaturetime history of each pixel and are stored in the form of 2D matrices and subsequently converted to images known as amplitude and phase image. Amplitude image focuses on the surface of a sample up to a limited depth and is suitable to offer the shape information. Phase image is less sensitive to shape information; independent of local disturbances such as non-uniform heating and emissivity; influenced by the depth attained by the thermal wave and is suitable for computation of defect depth [1]. The signal to noise ratio (SNR) of the amplitude and phase signals of detected defect features is essential for defect detection and characterization [2]. Thus, this study explores the fusion of amplitude and phase images to enhance the SNR of defect features, so the further accuracy of detectability can be improved. Pixel level data fusion based on Discrete wavelet transforms (DWT) technique is adopted in the study.

\section{Materials and methods}

Figure 1 shows the schematic illustration of a test sample made of Stainless steel (STS 304) considered in the study. The sample is square shaped with dimensions $180 \mathrm{~mm} \times 180 \mathrm{~mm} \times 10 \mathrm{~mm}$ and artificial defects of round cutouts with varying size and depth. The front side of the sample was painted black using KRYLON flat paint having an emissivity of 0.95 to create a uniform emissive surface. The experiment was conducted with a Lock-in system in reflection mode. A programmable function generator (Agilent 33210A, Malaysia) was used for the generation of sine waves. Two halogen lamps (OSRAM, Medium Flood, China) of $1 \mathrm{~kW}$ each were used as a heat source. The LIT system (Answer Tech, Republic of Korea) was used to synchronize the input and output signals. An IR camera (SC645, FLIR Systems, Sweden) with a $640 \times 480$ - pixel resolution and a wavelength of $7.5-13 \mu \mathrm{m}$ was used to record the thermal response of the sample. The schematic of the general test arrangement is shown in Figure 2.

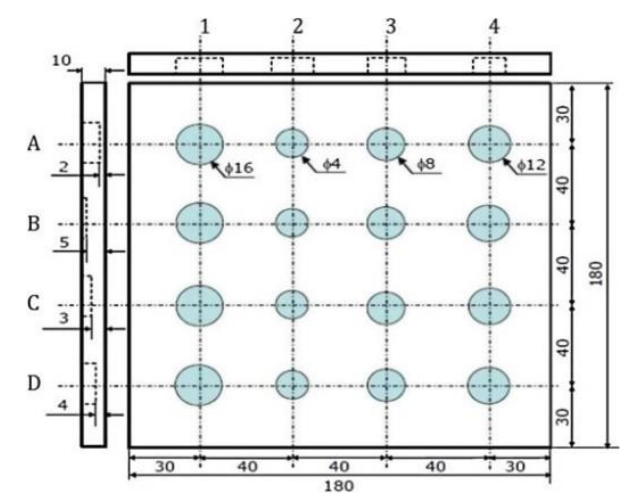

Fig. 1: STS 304 test sample with subsurface defects

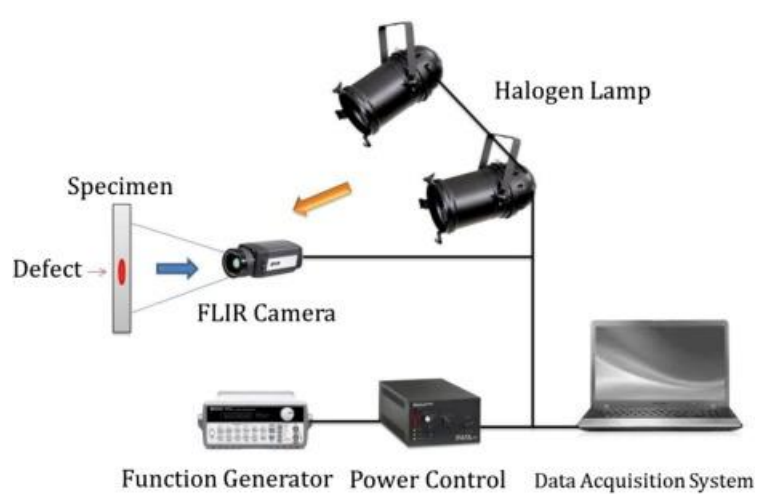

Fig. 2: Schematic of the experimental setup 


\section{Results and discussions}

The experimental data conducted at the modulation frequency of $0.02 \mathrm{~Hz}$ were considered in the study. The experiment was conducted for three complete excitation cycles. The thermal images from the second cycle were processed to compute the amplitude and phase of the thermal signal at all pixels using the Fourier transform [3]. Image fusion was performed on the amplitude and phase images as the input images. The basic Daubechies based DWT algorithm, as expressed in Eq. (1), was used for the fusion of input images. Fig. 3 shows the input amplitude and phase images, and fused images obtained with DWT technique.

$$
\mathrm{I}_{\mathrm{f}}(\mathrm{x}, \mathrm{y})=\mathrm{W}^{-1}\left[\varphi\left\{\mathrm{W}\left(\mathrm{I}_{1}(\mathrm{x}, \mathrm{y})\right), \mathrm{W}\left(\mathrm{I}_{2}(\mathrm{x}, \mathrm{y})\right)\right\}\right]
$$

where, $I_{f}$ is a fused image, $I_{1}$ and $I_{2}$ are the two images to fuse, $\varphi$ is the fusion rule, and $W$ and $W^{-1}$ are the DWT and IDWT, respectively.

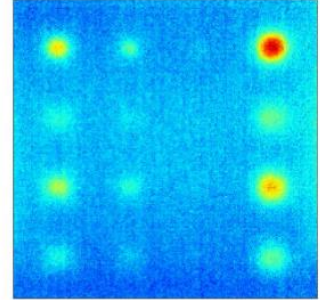

(a)

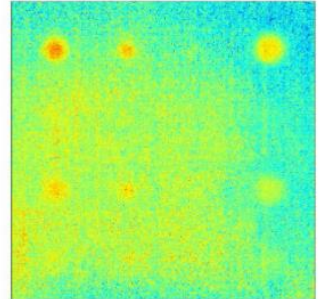

(b)

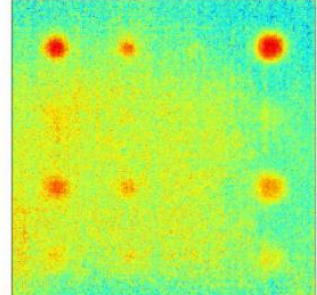

(c)

Fig. 3: Comparison of subsurface defect detection in (a) input amplitude image, (b) input phase image, and (c) output fused image with DWT technique at the excitation frequency $0.2 \mathrm{~Hz}$.

To evaluate the fusion performance of Eq. (1), the SNR of defects $A_{1}, A_{4}, C_{1}$, and $C_{4}$ at both the excitation frequencies were calculated and compared. Two ROls of $5 \times 5$ - pixel, one at the center of the defect and another in the adjacent sound region were considered for each selected defect. Then, the SNR was acquired by Eq. (2) [2].

$$
\mathrm{SNR}=20 \log _{10}\left(\frac{\left|\mathrm{DROI} \mathrm{I}_{\text {mean }}-\mathrm{SROI}_{\mathrm{mean}}\right|}{\sigma}\right)
$$

where $\mathrm{DROI}_{\text {mean }}$ is the arithmetic mean of all the pixels inside the inclusion region; $\mathrm{SROI}$ mean is the arithmetic mean of all the pixel inside the sound region and $\sigma$ is the standard deviation of the pixels inside the sound region. The corresponding results are listed in Table 1.

Table 1. Quantitative comparison of SNR between the fused and input images

\begin{tabular}{|c|c|c|c|}
\hline \multirow{2}{*}{ Defects ID } & \multicolumn{3}{|c|}{ SNR } \\
\cline { 2 - 4 } & Amplitude Image & Phase Image & Fused Image \\
\hline $\mathrm{A}_{1}$ & 21.20 & 16.43 & 22.85 \\
\hline $\mathrm{A}_{4}$ & 26.46 & 18.81 & 27.96 \\
\hline $\mathrm{C}_{1}$ & 21.62 & 14.00 & 23.11 \\
\hline $\mathrm{C}_{4}$ & 24.87 & 15.11 & 26.27 \\
\hline
\end{tabular}

\section{Conclusions}

This paper presented an innovative NDT\&E approach to combine amplitude and phase images to enhance the subsurface defects in STS 304 plate. DWT based pixel level data fusion method was adopted, and SNR was introduced to verify the robustness of the results. The resulting fused images showed that image fusion method improved the defects SNR as compared to using the phase image exclusively.

\section{References}

1. Shrestha Ranjit and Wontae Kim, "Non-destructive testing and evaluation of materials using active thermography and enhancement of signal to noise ratio through data fusion." Infrared Physics \& Technology, vol. 94, pp. 78-84 (2018).

2. Shrestha Ranjit, Jeonghak Park, and Wontae Kim, "Application of thermal wave imaging and phase shifting method for defect detection in Stainless steel." Infrared Physics \& Technology, vol. 76, pp. 676-683 (2016).

3. Ibarra-Castanedo Clemente and Xavier Maldague, "Pulsed phase thermography reviewed." Quantitative Infrared Thermography Journal, vol. 1, no. 1, pp. 47-70 (2004). 